\title{
THE 4.36-TH MOMENT OF THE RIEMANN ZETA-FUNCTION
}

\author{
MAKSYM RADZIWIEE
}

\begin{abstract}
Conditionally on the Riemann Hypothesis we obtain bounds of the correct order of magnitude for the $2 k$-th moment of the Riemann zeta-function for all positive real $k<2.181$. This provides for the first time an upper bound of the correct order of magnitude for some $k>2$; the case of $k=2$ corresponds to a classical result of Ingham [11. We prove our result by establishing a connection between moments with $k>2$ and the so-called "twisted fourth moment". This allows us to appeal to a recent result of Hughes and Young 10. Furthermore we obtain a point-wise bound for $\left|\zeta\left(\frac{1}{2}+\mathrm{i} t\right)\right|^{2 r}$ (with $0<r<1$ ) that can be regarded as a multiplicative analogue of Selberg's bound for $S(T)$ [18] . We also establish asymptotic formulae for moments $(k<2.181)$ slightly off the half-line.
\end{abstract}

\section{InTRODUCTION.}

An important problem in analytic number theory is to gain an understanding of the moments of the Riemann zeta-function,

$$
M_{k}(T):=\int_{T}^{2 T}\left|\zeta\left(\frac{1}{2}+\mathrm{i} t\right)\right|^{2 k} \mathrm{~d} t .
$$

It is conjectured that $M_{k}(T) \sim C_{k} \cdot T(\log T)^{k^{2}}$ for all $k>0$. Recent evidence for this conjecture comes from many sources. Classical analytical arguments for $k=6$ and $k=8$ (see [1] and [2]), models based on random matrix theory (see [15] and [6]), and considerations of multiple Dirichlet series (see [5]) ... have all led to the same conjecture for $M_{k}(T)$.

Following the work of Hardy-Littlewood [7, and Ingham [11, this asymptotic formulae is known for $k=0,1,2$. Lower bounds of the correct order of magnitude have been established for $2 k \in \mathbb{N}$ by Ramachandra [17], for $2 k \in \mathbb{Q}$ by Heath-Brown [8], and recently for all $2 k>0$ by the author and Soundararajan [16].

Less is known when it comes to upper bounds for $M_{k}(T)$. Assuming the Riemann Hypothesis Heath-Brown [8] established an upper bound of the correct order of magnitude for $M_{k}(T)$ for $0 \leqslant k<2$. For $k>2$, we have Soundararajan's [19] upper bound,

$$
M_{k}(T) \ll_{k, \varepsilon} T(\log T)^{k^{2}+\varepsilon}
$$

(Here $\varepsilon=O(1 / \log \log \log T)$ as remarked by Ivić [12]). While these results come close to the expected order of growth of $M_{k}(T)$, they miss the correct order of magnitude of $M_{k}(T)$ for all $k>2$. In this paper, we develop a new method which obtains on the Riemann Hypothesis an upper bound of the right order of magnitude for the $2 k$-th moment of $\zeta(s)$ in the range $2<k<2+\frac{2}{11}$.

Theorem 1. Assume the Riemann Hypothesis. If $0<k<2+\frac{2}{11}$, then,

$$
M_{k}(T) \ll_{k} T(\log T)^{k^{2}} .
$$

2000 Mathematics Subject Classification. Primary: 11M06, Secondary: 11M50.

The author is partially supported by a NSERC PGS-D award. 
Our method also provides a new way to obtain Heath-Brown's [8] bounds in the range $0<k<2$. One surprising feature (which is also present in [8]) is that the implicit constant $c(k)$ in our bounds for $M_{k}(T)$ tends to infinity as $k \rightarrow 2^{-}$even though we have Ingham's asymptotic formulae for $k=2$ and now also good upper bounds for $2<k<2+\frac{2}{11}$. In particular $c(k)$ stays bounded as $k \rightarrow 2^{+}$from the left.

As a quick application of Theorem 1, we obtain asymptotic formulae for moments $(k<$ $\left.2+\frac{2}{11}\right)$ slightly off the half-line. These are known for all $\sigma \geqslant \frac{1}{2}$ for $k=1,2$ by the classical work of Hardy-Littlewood [7] and Ingham [11] and following Soundararajan [19] we can also establish asymptotic formulae for all $k>0$ when $\sigma \geqslant \frac{1}{2}+c_{k} \cdot \log \log T / \log T$. However, as shown below, we can do better in terms of $\sigma$ for moments with $k<2+\frac{2}{11}$.

Corollary 1. Assume the Riemann Hypothesis. Let $\psi(T)$ be such that $\psi(T) \rightarrow \infty$ arbitrarily slowly and $\psi(T)=o(\log T)$. Set $\sigma=\frac{1}{2}+\psi / \log T$. If $0<k<2+\frac{2}{11}$, then,

$$
\int_{T}^{2 T}|\zeta(\sigma+\mathrm{i} t)|^{2 k} \mathrm{~d} t=\sum_{n \geq 1} \frac{d_{k}(n)^{2}}{n^{2 \sigma}} \cdot\left(1+O\left(e^{-\psi / 100}\right)\right) .
$$

Returning to Theorem 1, a natural line of attack is to consider the so-called "twisted fourth moment"

$$
\int_{T}^{2 T}\left|\zeta\left(\frac{1}{2}+\mathrm{i} t\right)\right|^{4} \cdot\left|A\left(\frac{1}{2}+\mathrm{i} t\right)\right|^{2} \mathrm{~d} t
$$

with $A(\cdot)$ a Dirichlet polynomial. The expression in (3) was first considered by Iwaniec in [13] and then Deshouillers and Iwaniec in [4] and [3]. They obtained a bound $T^{1+\varepsilon}$ for Dirichlet polynomials $A(s)$ of length $T^{1 / 5-\varepsilon}$ and with bounded coefficients (say). Their work has been substantially refined by Watt [20], who allows for Dirichlet polynomials of length up to $T^{1 / 4-\varepsilon}$. An asymptotic formulae for (3) has been recently developed by Hughes and Young [10] (see also related work by Jara [14]) and this forms one of the key ingredients in our proof.

Until now the known results about (3) had no consequence on (19) even on the assumption of the Riemann Hypothesis; the main difficulty being the absence of a bound for $\left|\zeta\left(\frac{1}{2}+\mathrm{i} t\right)\right|^{2 r}$ $(0<r<1)$ in terms of a short Dirichlet polynomial. Our proof of Theorem 1 provides such a connection for all $2<k<3$.

Corollary 2. Assume the Riemann Hypothesis. Let $0<r<1$. Then,

$$
M_{2+r}(T) \ll \int_{T}^{2 T}\left|\zeta\left(\frac{1}{2}+\mathrm{i} t\right)\right|^{4} \cdot\left|\sum_{n \leqslant x} \frac{d_{r}(n) W(n)}{n^{\frac{1}{2}+\mathrm{i} t}}\right|^{2} \mathrm{~d} t
$$

where $x=T^{r / 2+2 \delta}<T$ and $\delta>0$ is an arbitrary but fixed positive real number, and $W(\cdot)$ is a continuous smoothing, defined as

$$
W(n):=\left\{\begin{array} { l l } 
{ 1 } & { \text { if } n \leqslant y } \\
{ \frac { \operatorname { l o g } ( x / n ) } { \operatorname { l o g } ( x / y ) } } & { \text { if } y < n \leqslant x } \\
{ 0 } & { \text { if } n > x }
\end{array} \text { where } \left\{\begin{array}{l}
x=T^{r / 2+2 \delta} \\
y=T^{r / 2+\delta}
\end{array}\right.\right.
$$

The implicit constant in (4) depends at most on $r$ and $\delta$.

The main idea in our proof is to obtain bounds for $|\zeta(s)|^{2 r}(0<r<1)$ in terms of a Dirichlet polynomial and we do this in a way remniscent of Selberg's work on $S(T)$ [18]. 
More precisely, we write

$$
\zeta(s)^{r}=\sum_{n \leqslant x} \frac{d_{r}(n) W(n)}{n^{s}}+O\left(\frac{y^{\frac{1}{2}-\sigma}}{\log (x / y)} \int_{-\infty}^{\infty} \frac{\left|\zeta\left(\frac{1}{2}+\mathrm{i} t+\mathrm{i} v\right)\right|^{r}}{\left(\sigma-\frac{1}{2}\right)^{2}+v^{2}} \mathrm{~d} v\right)
$$

and upon averaging over $T \leqslant t \leqslant 2 T$ we bound the contribution from the error term in terms of the moment itself times a small constant $<1$. We provide below a variant of this idea which might of independent interest.

Proposition 1. Assume the Riemann Hypothesis. Let $0<r \leqslant 1$ and $0<\delta<1$ such that $r / 2+3 \delta<1$ be given. Then, for $T \leqslant t \leqslant 2 T$ with $T \geqslant T_{0}$ (and $T_{0}$ a large absolute constant),

$$
\left|\zeta\left(\frac{1}{2}+\mathrm{i} t\right)\right|^{2 r} \leqslant 3 e^{12 r / \delta} \int_{\mathbb{R}}\left|\sum_{n \leqslant x} \frac{d_{r}(n) W(n)}{n^{2 \sigma_{0}-\frac{1}{2}+\mathrm{i} t+\mathrm{i} v}}\right|^{2} \cdot \frac{(1 / \pi)\left(\sigma_{0}-\frac{1}{2}\right) \mathrm{d} v}{\left(\sigma_{0}-\frac{1}{2}\right)^{2}+v^{2}}+O\left(\frac{1}{T}\right)
$$

where $x=T^{r / 2+2 \delta}$ and $\sigma_{0}=\frac{1}{2}+\frac{(4 / \delta)}{\log T}$.

Integrating the above inequality over $T \leqslant t \leqslant 2 T$ we recover Heath-Brown's [8] upper bound $M_{r}(T) \ll T(\log T)^{r^{2}}$ for all $0 \leqslant r<2$. Furthermore, Proposition 1 allows us to generalize (44) to a product of two (or more!) Dirichlet polynomials and also to obtain bounds of the correct order of magnitude for the "twisted $r$-th moment" $\int_{T}^{2 T}\left|\zeta\left(\frac{1}{2}+\mathrm{i} t\right)\right|^{2 r} \cdot\left|A\left(\frac{1}{2}+\mathrm{i} t\right)\right|^{2} \mathrm{~d} t$ for $r<2$ and Dirichlet polynomials of length $<T^{1-r / 2-\varepsilon}$ and for $2<r<2+\frac{2}{11}$ and Dirichlet polynomials of length $T^{1 / 11-r / 2-\varepsilon}$ when combined with Hughes and Young's work [10].

The appearance of $2 \sigma_{0}-\frac{1}{2}+$ it in (7) is inessential : we can further bound the right-hand side by

$$
\leqslant C(\varepsilon) \int_{-\infty}^{\infty}\left|\sum_{n \leqslant x} \frac{d_{r}(n) W(n)}{n^{\frac{1}{2}+\mathrm{i} t+\mathrm{i} v}}\right|^{2} \cdot \frac{(1 / \pi)\left(\sigma_{0}-\frac{1}{2}\right) \mathrm{d} v}{\left(\sigma_{0}-\frac{1}{2}\right)^{2}+v^{2}},
$$

if we wish to do so. It is also worth noticing that the error term $O(1 / T)$ in (17) is always smaller than the main term.

Proposition 1 can be regarded as a multiplicative analogue of Soundararajan's [19] upper bound for $\log \left|\zeta\left(\frac{1}{2}+\mathrm{i} t\right)\right|$,

$$
\log \left|\zeta\left(\frac{1}{2}+\mathrm{i} t\right)\right| \leqslant \mathfrak{R} \sum_{n \leqslant x} \frac{\Lambda(n) / \log n}{n^{\frac{1}{2}+\frac{\lambda}{\log x}+\mathrm{i} t}} \cdot \frac{\log (x / n)}{\log x}+\frac{1+\lambda}{2} \cdot \frac{\log T}{\log x}+O\left(\frac{1}{\log x}\right)
$$

where $T \leqslant t \leqslant 2 T$. Note however, the greater degree of flexibility in the choice of parameters $1 \leqslant x \leqslant T^{2}$ and $\lambda \geqslant \lambda_{0}=0.4912 \ldots$ in the inequality above.

Besides Selberg's work on $S(T)$ our proof is also inspired by ideas from Ramachandra's and Heath-Brown's work. Our proof adapts to the case of central moments of $L$-functions (giving for example an alternative proof of the result in [9] in the range $0<k<2$ ) and we plan to return to this subject on a later occasion.

Acknowledgment. I would like to thank my supervisor Prof. Soundararajan for his advice and a simplification of my original proof, and Prof. Matt Young for a number of insightful remarks.

Notation. We denote by $\varepsilon$ an arbitrarily small but fixed constant, not necessarily the same from line to line and by $T_{0}$ a large absolute constant. We will write $s=\sigma+\mathrm{i} t$. 


\section{KEY IDEAS}

The relevance of the following lemma to moments of the Riemann zeta-function (on the assumption of the Riemann Hypothesis) was first pointed out by Soundararajan in [19].

Lemma 1. Let $\xi(s):=s(s-1) \pi^{-s / 2} \Gamma(s / 2) \zeta(s)$. The Riemann Hypothesis holds if and only if for every fixed $t,|\xi(\sigma+\mathrm{i} t)|$ is an increasing function of $\sigma$ for $\sigma \geqslant \frac{1}{2}$. In particular on the Riemann Hypothesis, uniformly in $\sigma^{\prime} \geqslant \sigma \geqslant \frac{1}{2}$,

$$
|\zeta(\sigma+\mathrm{i} t)|^{2 r} \leqslant T^{r\left(\sigma^{\prime}-\sigma\right)} \cdot\left|\zeta\left(\sigma^{\prime}+\mathrm{i} t\right)\right|^{2 r},
$$

for all $T \leqslant t \leqslant 2 T$ with $T \geqslant T_{0}$.

Proof. We have the following Hadamard factorization formula,

$$
\xi(s)=\xi(0) \prod_{\rho}\left(1-\frac{s}{\rho}\right)
$$

where we group together the zeroes $\rho$ and $\bar{\rho}$ to ensure the convergence of the product. If the Riemann Hypothesis holds, then $\Re(\rho)=\frac{1}{2}$ for all $\rho$ 's, and hence if $\Re s \geqslant \frac{1}{2}$ and $c>0$, then

$$
|\rho-s| \leqslant|\rho-(s+c)|
$$

for any $\rho$, because the distance of $s+c$ from any given point on the line $\mathfrak{R} z=\frac{1}{2}$ is always greater than that of $s$. Thus

$$
\left|\frac{\xi(s)}{\xi(s+c)}\right| \leqslant \prod_{\rho}\left|\frac{\rho-s}{\rho-(s+c)}\right| \leqslant 1,
$$

as claimed. To establish the converse suppose to the contrary that the Riemann Hypothesis is false, but $|\xi(\sigma+\mathrm{i} t)|$ is still an increasing function of $\sigma$. Let $\rho=\sigma^{\prime}+$ it denote a zero of $\zeta(s)$ off the half-line. Pick an $\frac{1}{2} \leqslant \sigma<\sigma^{\prime}$ for which $|\xi(\sigma+\mathrm{i} t)|>0$; such a $\sigma$ exists because $\xi$ is analytic and not identically zero. Since $|\xi(\sigma+\mathrm{i} t)|$ is increasing $0<|\xi(\sigma+\mathrm{i} t)| \leqslant\left|\xi\left(\sigma^{\prime}+\mathrm{i} t\right)\right|=0$ and this is a contradiction.

Finally, if the Riemann Hypothesis holds, then the inequality $|\zeta(\sigma+\mathrm{i} t)| \leqslant T^{\left(\sigma^{\prime}-\sigma\right)} \cdot\left|\zeta\left(\sigma^{\prime}+\mathrm{i} t\right)\right|$ follows from $|\xi(\sigma+\mathrm{i} t)| \leqslant\left|\xi\left(\sigma^{\prime}+\mathrm{i} t\right)\right|$ upon unfolding $\xi(s)$ into $s(s-1) \pi^{-s / 2} \Gamma(s / 2) \zeta(s)$ and using Stirling's formula.

Lemma 2. Assume the Riemann Hypothesis and let $W(n)$ be defined as in (5). Then, for any fixed $r>0$, uniformly in $\frac{1}{2} \leqslant \sigma \leqslant 1, T \leqslant t \leqslant 2 T$ (with $T \geqslant T_{0}$ ) and $1 \leqslant y \leqslant x / 2 \leqslant T^{1-\varepsilon}$,

$$
\zeta(s)^{r}=\sum_{n \geqslant 1} \frac{d_{r}(n) W(n)}{n^{s}}+\frac{\theta y^{\frac{1}{2}-\sigma}}{\log (x / y)} \cdot \frac{1}{\pi} \int_{-\infty}^{\infty} \frac{\left|\zeta\left(\frac{1}{2}+\mathrm{i} t+\mathrm{i} v\right)\right|^{r}}{\left(\sigma-\frac{1}{2}\right)^{2}+v^{2}} \cdot \mathrm{d} v+O\left(\frac{1}{T}\right),
$$

with a $\theta$ of absolute value at most one.

Proof. Consider

$$
\sum_{n \geqslant 1} \frac{d_{r}(n) W(n)}{n^{s}}=\frac{1}{2 \pi \mathrm{i}} \int_{(c)} \zeta(s+w)^{r} \cdot \frac{x^{w}-y^{w}}{w^{2} \cdot \log (x / y)} \mathrm{d} w
$$

with $c=1+1 / \log T$. In order to avoid a branch cut emanating from $w=1-s$, we take out the little piece corresponding to $|\mathfrak{I}(w-s)| \leqslant \varepsilon$ from the integral above, making an error of $O(1 / T)$. Shifting contours in the remaining two integrals, we obtain $\zeta(s)^{r}$ from a pole at 
$w=0$ and an error of $O(1 / T)$ from the integrals over horizontal lines, since $|\zeta(s)| \ll T^{\varepsilon}$ for $\sigma \geqslant \frac{1}{2}$ by Lindelöf's Hypothesis. Thus,

$$
\sum_{n \geqslant 1} \frac{d_{r}(n) W(n)}{n^{s}}=\zeta(s)^{r}+\frac{1}{2 \pi \mathrm{i}} \int_{\mathcal{L}} \zeta(s+w)^{r} \cdot \frac{\left(x^{w}-y^{w}\right) \mathrm{d} w}{w^{2} \cdot \log (x / y)}+O\left(\frac{1}{T}\right) .
$$

where $\mathcal{L}$ corresponds to the line $\mathfrak{R} s=\frac{1}{2}-\sigma$ without the segment with $|\mathfrak{I}(w-s)| \leqslant \varepsilon$. Since the integral on the right is in absolute value at most

$$
\frac{\theta y^{\frac{1}{2}-\sigma}}{\log (x / y)} \cdot \frac{1}{\pi} \int_{-\infty}^{\infty} \frac{\left|\zeta\left(\frac{1}{2}+\mathrm{i} t+\mathrm{i} v\right)\right|^{r}}{\left(\sigma-\frac{1}{2}\right)^{2}+v^{2}} \cdot \mathrm{d} v
$$

the lemma follows.

Lemma 3. Assume the Riemann Hypothesis. Then uniformly in $\frac{1}{2}<\sigma \leqslant 1$ and $T \leqslant t \leqslant 2 T$ with $T \geqslant T_{0}$,

$\int_{T}^{2 T}\left|\zeta\left(\frac{1}{2}+\mathrm{i} t\right)\right|^{4} \cdot\left(\int_{-\infty}^{\infty} \frac{\left|\zeta\left(\frac{1}{2}+\mathrm{i} t+\mathrm{i} v\right)\right|^{r}}{\left(\sigma-\frac{1}{2}\right)^{2}+v^{2}} \cdot \mathrm{d} v\right)^{2} \mathrm{~d} t \leqslant \frac{2 \pi^{2}}{\left(\sigma-\frac{1}{2}\right)^{2}} \int_{T}^{2 T}\left|\zeta\left(\frac{1}{2}+\mathrm{i} t\right)\right|^{2 k} \cdot \mathrm{d} t+O\left(T^{1-\varepsilon}\right)$,

where $2 k=4+2 r$.

Proof. The left-hand side is by Cauchy's inequality at most,

$$
\begin{aligned}
& \frac{\pi}{\sigma-\frac{1}{2}} \int_{T}^{2 T}\left|\zeta\left(\frac{1}{2}+\mathrm{i} t\right)\right|^{4} \cdot \int_{-\infty}^{\infty} \frac{\left|\zeta\left(\frac{1}{2}+\mathrm{i} t+\mathrm{i} v\right)\right|^{2 r}}{\left(\sigma-\frac{1}{2}\right)^{2}+v^{2}} \mathrm{~d} v \mathrm{~d} t \\
= & \frac{\pi}{\sigma-\frac{1}{2}} \int_{-\infty}^{\infty} \int_{T}^{2 T}\left|\zeta\left(\frac{1}{2}+\mathrm{i} t\right)\right|^{4} \cdot\left|\zeta\left(\frac{1}{2}+\mathrm{i} t+\mathrm{i} v\right)\right|^{2 r} \mathrm{~d} t \cdot \frac{\mathrm{d} v}{\left(\sigma-\frac{1}{2}\right)^{2}+v^{2}} .
\end{aligned}
$$

The inner integrand is at most $\left|\zeta\left(\frac{1}{2}+\mathrm{i} t\right)\right|^{4+2 r}+\left|\zeta\left(\frac{1}{2}+\mathrm{i} t+\mathrm{i} v\right)\right|^{4+2 r}$. The first term contributes in total $\left(\pi /\left(\sigma-\frac{1}{2}\right)\right)^{2} \int_{T}^{2 T}\left|\zeta\left(\frac{1}{2}+\mathrm{i} t\right)\right|^{4+2 r} \mathrm{~d} t$, while the second makes a contribution of

$$
\leqslant \frac{\pi}{\sigma-\frac{1}{2}} \int_{-\infty}^{\infty} \int_{T}^{2 T}\left|\zeta\left(\frac{1}{2}+\mathrm{i} t+\mathrm{i} v\right)\right|^{4+2 r} \mathrm{~d} t \cdot \frac{\mathrm{d} v}{\left(\sigma-\frac{1}{2}\right)^{2}+v^{2}}
$$

Consider the integral over $v$. By Lindelöf's Hypothesis the integral over $t$ is bounded by $T(T+|v|)^{\varepsilon}$. Therefore the terms with $|v| \geqslant T^{1-\varepsilon}$ contribute $\ll T^{\varepsilon}$ due to the rapid decay of the kernel $\left(\left(\sigma-\frac{1}{2}\right)^{2}+v^{2}\right)^{-1}$. On the other hand the contribution from the terms $|v| \leqslant T^{1-\varepsilon}$ is bounded by

$$
\int_{-T^{1-\varepsilon}}^{T^{1-\varepsilon}} \int_{T-T^{1-\varepsilon}}^{2 T+T^{1-\varepsilon}}\left|\zeta\left(\frac{1}{2}+\mathrm{i} t\right)\right|^{2 k} \cdot \mathrm{d} t \cdot \frac{\mathrm{d} v}{\left(\sigma-\frac{1}{2}\right)^{2}+v^{2}} \leqslant \frac{\pi}{\sigma-\frac{1}{2}} \int_{T}^{2 T}\left|\zeta\left(\frac{1}{2}+\mathrm{i} t\right)\right|^{2 k} \mathrm{~d} t+O\left(T^{1-\varepsilon}\right) .
$$

Combining these bounds we obtain the claim.

\section{The work of Hughes And Young.}

The main result (Theorem 1) in Hughes and Young paper [10] holds unconditionally. However, we'll aid ourselves in the proof of the next lemma by assuming the Riemann Hypothesis. 
Lemma 4. Assume the Riemann Hypothesis. Let $W(n)$ denote the coefficients in (5). If $0 \leqslant x \leqslant T^{1 / 11-\varepsilon}$ then,

$$
\int_{T}^{2 T}\left|\zeta\left(\frac{1}{2}+\mathrm{i} t\right)\right|^{4} \cdot\left|\sum_{n \leqslant x} \frac{d_{r}(n) W(n)}{n^{\frac{1}{2}+\mathrm{i} t}}\right|^{2} \mathrm{~d} t \ll T(\log T)^{(2+r)^{2}},
$$

and where the implicit constant depends at most on $\varepsilon$ and $r$.

Proof. We will use freely the notation used in Theorem 1 of Hughes and Young's paper [10]. We pick a smooth function $g$ with $g(t)=1$ in $[T ; 2 T], g(t) \leqslant 1$ elsewhere, $g^{(j)}(t) \ll T^{-j(1-\varepsilon)}$ for all $j \geqslant 0$ and with support contained in $[T / 2 ; 3 T]$. By Lemma 1 the left-hand side of (8) is

$$
\ll \int_{T}^{2 T}\left|\zeta\left(\frac{1}{2}+\alpha+\mathrm{i} t\right)\right|^{2} \cdot\left|\zeta\left(\frac{1}{2}+\beta+\mathrm{i} t\right)\right|^{2} \cdot\left|\sum_{n \leq x} \frac{d_{r}(n) W(n)}{n^{\frac{1}{2}+\mathrm{i} t}}\right|^{2} \cdot g(t) \mathrm{d} t
$$

where $\alpha=1 / \log T$ and $\beta=2 / \log T$. By the main result of [10] for $(m, n)=1$,

$$
\int_{\mathbb{R}}\left(\frac{m}{n}\right)^{\mathrm{i} t} \cdot\left|\zeta\left(\frac{1}{2}+\alpha+\mathrm{i} t\right)\right|^{2} \cdot\left|\zeta\left(\frac{1}{2}+\beta+\mathrm{i} t\right)\right|^{2} g(t) \mathrm{d} t=\int_{\mathbb{R}} g(t) \mathrm{d} t \cdot \frac{Z_{\alpha, \beta, \alpha, \beta, m, n}(0)}{\sqrt{m n}}+\ldots+\mathcal{E},
$$

where $\mathcal{E} \ll T^{3 / 4+\varepsilon} \cdot(m n)^{7 / 8}$ and inside ... we omitted a sum of five more additional terms resembling the expression displayed above (the only essential difference being that instead of $Z_{\alpha, \beta, \alpha, \beta, m, n}(0)$ we will have $Z_{\alpha^{\prime}, \beta^{\prime}, \gamma^{\prime}, \delta^{\prime}, m, n}(0)$ with $\alpha^{\prime}, \beta^{\prime}, \gamma^{\prime}, \delta^{\prime} \in\{ \pm \alpha, \pm \beta\}$. Our choice of $\alpha, \beta$ ensures that each of these five $Z_{\alpha^{\prime}, \beta^{\prime}, \gamma^{\prime}, \delta^{\prime}, m, n}(0)$ is at most $C(\log T)^{4}$ times a multiplicative function of $(m, n))$.

This being said, the left-hand side of (8) is

$$
\begin{aligned}
& \ll \int_{\mathbb{R}}\left|\zeta\left(\frac{1}{2}+\alpha+\mathrm{i} t\right)\right|^{2} \cdot\left|\zeta\left(\frac{1}{2}+\beta+\mathrm{i} t\right)\right|^{2} \cdot\left|\sum_{n \leqslant x} \frac{d_{r}(n) W(n)}{n^{\frac{1}{2}+\mathrm{i} t}}\right|^{2} g(t) \mathrm{d} t \\
& =\sum_{m, n \leqslant x} \frac{d_{r}(n) W(n) d_{r}(m) W(m)}{\sqrt{m n}} \int_{\mathbb{R}}\left(\frac{m}{n}\right)^{\mathrm{i} t} \cdot\left|\zeta\left(\frac{1}{2}+\alpha+\mathrm{i} t\right)\right|^{2} \cdot\left|\zeta\left(\frac{1}{2}+\beta+\mathrm{i} t\right)\right|^{2} g(t) \mathrm{d} t \\
& =\sum_{m, n \leq x} \frac{d_{r}(m) d_{r}(n) W(m) W(n)}{\sqrt{m n}} \cdot \frac{(m, n)}{\sqrt{m n}} \int_{\mathbb{R}} g(t) \mathrm{d} t \cdot Z_{\alpha, \beta, \alpha, \beta, m^{\prime}, n^{\prime}}(0)+\ldots+\mathcal{E}^{\prime},
\end{aligned}
$$

where $m^{\prime}:=m /(m, n), n^{\prime}:=n /(m, n)$ and $\mathcal{E}^{\prime} \ll T^{1-\varepsilon}$. The function $Z_{\alpha, \beta, \alpha, \beta, m^{\prime}, n^{\prime}}(0)$ factors into

$$
Z_{\alpha, \beta, \alpha, \beta, m^{\prime}, n^{\prime}}(0)=A_{\alpha, \beta, \alpha, \beta}(0) \cdot B_{\alpha, \beta, \alpha, \beta, m^{\prime}, n^{\prime}}(0) \ll(\log T)^{4} \cdot\left|B_{\alpha, \beta, \alpha, \beta, m^{\prime}, n^{\prime}}(0)\right|
$$

and $B_{\alpha, \beta, \alpha, \beta, m^{\prime}, n^{\prime}}(0)$ is multiplicative in the two variables $(m, n)$. We bound the resulting sum over $m, n$ in (91) (using $0 \leqslant W(n) \leqslant 1$ for $n \leqslant x$ ) by

$$
\begin{aligned}
& \leqslant \sum_{m, n \leqslant x} \frac{d_{r}(m) d_{r}(n)}{[m, n]} \cdot\left|B_{\alpha, \beta, \alpha, \beta, m^{\prime}, n^{\prime}}(0)\right| \\
& \leqslant \prod_{p \leqslant x}\left(1+\frac{r}{p} \cdot\left|\sigma_{\alpha, \beta}(p)\right|+\frac{r}{p} \cdot\left|\sigma_{\alpha, \beta}(p)\right|+\frac{r^{2}}{p}+O\left(\frac{1}{p^{2}}\right)\right) \\
& \leqslant \prod_{p \leqslant x}\left(1+\frac{r}{p^{1+\Re \alpha}}+\frac{r}{p^{1+\Re \beta}}+\frac{r}{p^{1+\Re \alpha}}+\frac{r}{p^{1+\Re \beta}}+\frac{r^{2}}{p}+O\left(\frac{1}{p^{2}}\right)\right) .
\end{aligned}
$$


We can bound the sum over $m, n \leqslant x$ by an truncated Euler product because the summand is a multiplicative function in the two variable $(m, n)$ (i.e $f\left(m_{1} n_{1}, m_{2} n_{2}\right)=f\left(m_{1}, m_{2}\right) f\left(n_{1}, n_{2}\right)$ for $\left.\left(m_{1} m_{2}, n_{1} n_{2}\right)=1\right)$. Since

$$
\sum_{p \leqslant x} \frac{1}{p^{1+\alpha}} \leqslant \log \log x+O(1) \text { for } \alpha \ll 1 / \log x,
$$

the expression in (10) is $\ll(\log x)^{r^{2}+4 r} \ll(\log T)^{r^{2}+4 r}$. Thus the displayed expression in (9) is $\ll T(\log T)^{4+r^{2}+4 r}=T(\log T)^{(2+r)^{2}}$. The computation of the five additional terms in ... is similar and each of them contributes $\ll T(\log T)^{(2+r)^{2}}$. We conclude that (9) is $\ll T(\log T)^{(2+r)^{2}}$ as desired.

\section{Proof of Theorem 1 (and Corollary 21)}

Proof. Theorem [1 is known for $k<2$, by the work of Heath-Brown [8], and for $k=2$ as a consequence of Ingham's [11] asymptotic formulae. We thus write $k=2+r$, with $0<r<2 / 11$. By Lemma 1 ,

$$
M_{k}(T)=\int_{T}^{2 T}\left|\zeta\left(\frac{1}{2}+\mathrm{i} t\right)\right|^{4} \cdot\left|\zeta\left(\frac{1}{2}+\mathrm{i} t\right)\right|^{2 r} \mathrm{~d} t \leqslant T^{r\left(\sigma-\frac{1}{2}\right)} \int_{T}^{2 T}\left|\zeta\left(\frac{1}{2}+\mathrm{i} t\right)\right|^{4} \cdot|\zeta(\sigma+\mathrm{i} t)|^{2 r} \mathrm{~d} t .
$$

By Lemma 2 applied to $\zeta\left(\frac{1}{2}+\mathrm{i} t\right)^{r}$ and the inequality $|a+b|^{2} \leqslant 2|a|^{2}+2|b|^{2}$ the above is at most

$$
\begin{aligned}
& \leqslant 2 T^{r\left(\sigma-\frac{1}{2}\right)} \int_{T}^{2 T}\left|\zeta\left(\frac{1}{2}+\mathrm{i} t\right)\right|^{4} \cdot\left|\sum_{n \leqslant x} \frac{d_{r}(n) W(n)}{n^{\frac{1}{2}+\mathrm{i} t}}\right|^{2} \mathrm{~d} t+ \\
& +2 T^{r\left(\sigma-\frac{1}{2}\right)} \cdot \frac{y^{1-2 \sigma}}{\log ^{2}(x / y)} \cdot \frac{1}{\pi^{2}} \int_{T}^{2 T}\left|\zeta\left(\frac{1}{2}+\mathrm{i} t\right)\right|^{4} \cdot\left(\int_{-\infty}^{\infty} \frac{\left|\zeta\left(\frac{1}{2}+\mathrm{i} t+\mathrm{i} v\right)\right|^{r}}{\left(\sigma-\frac{1}{2}\right)^{2}+v^{2}} \mathrm{~d} v\right)^{2} \mathrm{~d} t
\end{aligned}
$$

plus a negligible contribution from $O(1 / T)$, which we henceforth omit. By Lemma 4 the first term in (11) is

$$
\leqslant C \cdot T^{r\left(\sigma-\frac{1}{2}\right)} \cdot T(\log T)^{k^{2}},
$$

provided that $0 \leqslant x \leqslant T^{1 / 11-\varepsilon^{\prime}}$ and with a finite constant $C=C\left(\varepsilon^{\prime}, r\right)$ depending only on $\varepsilon^{\prime}$ and $r$. By Lemma 3 the second term in (11) is at most

$$
2 T^{r\left(\sigma-\frac{1}{2}\right)} \cdot \frac{y^{1-2 \sigma}}{\log ^{2}(x / y)} \cdot \frac{2 M_{k}(T)}{\left(\sigma-\frac{1}{2}\right)^{2}}+O(T) .
$$

One among many possible choices for $x, y$ and $\sigma$ is given by first fixing an $\delta>0$ such that $r / 2+3 \delta<1 / 11$, and then setting $x=T^{r / 2+2 \delta}, y=T^{r / 2+\delta}$, and $\sigma-1 / 2=(2 / \delta) / \log T$. Note that $x \leqslant T^{1 / 11-\delta}$ so that Lemma 4 is applicable and also that (12) is $\leqslant C(\delta, r) e^{2 r / \delta} \cdot T(\log T)^{k^{2}}$ by our choice of $\sigma$. In the main term in (13) we obtain,

$$
2 e^{2 r / \delta} \cdot \frac{e^{-4(r / 2+\delta) / \delta}}{\log ^{2}\left(T^{\delta}\right)} \cdot \frac{2 M_{k}(T)}{(2 / \delta)^{2}(\log T)^{-2}}=e^{-4} \cdot M_{k}(T)<\frac{M_{k}(T)}{2} .
$$

Therefore $M_{k}(T) \leqslant C(\delta, r) e^{2 r / \delta} T(\log T)^{k^{2}}+M_{k}(T) / 2+O(T)$ and hence $M_{k}(T) \ll T(\log T)^{k^{2}}$ as claimed. In order to obtain Corollary 2 it suffices not to bound the first term in (11) by (12) and instead leave it as it is. 


\section{Proof of Corollary 1$]$}

Proof. Fix an $\delta>1 / 100$ (with $\delta<2 / 100$ ) and in Lemma 2 choose $x=T^{2 \delta}, y=T^{\delta}, r=k$ and $\sigma=\frac{1}{2}+\psi / \log T$. Using the inequality $\left|a+e^{-\delta \psi} b\right|^{2}=|a|^{2} \cdot\left(1+O\left(e^{-\delta \psi}\right)\right)+O\left(e^{-\delta \psi}|b|^{2}\right)$ we get,

$\left|\zeta(\sigma+\mathrm{i} t)^{k}\right|^{2}=\left|\sum_{n \leqslant x} \frac{d_{k}(n) W(n)}{n^{\sigma+\mathrm{i} t}}\right|^{2}\left(1+O\left(e^{-\delta \psi}\right)\right)+O\left(\frac{e^{-\delta \psi}}{\log ^{2}(T)}\left(\int_{-\infty}^{\infty} \frac{\left|\zeta\left(\frac{1}{2}+\mathrm{i} t+\mathrm{i} v\right)\right|^{k}}{\left(\sigma-\frac{1}{2}\right)^{2}+v^{2}} \mathrm{~d} v\right)^{2}\right)$

plus a negligible contribution $\left(\ll T^{-2}\right)$ from $O(1 / T)$. Hence,

$$
\begin{aligned}
\int_{T}^{2 T}|\zeta(\sigma+\mathrm{i} t)|^{2 k} \mathrm{~d} t= & \int_{T}^{2 T}\left|\sum_{n \leqslant x} \frac{d_{k}(n) W(n)}{n^{\sigma+\mathrm{i} t}}\right|^{2} \mathrm{~d} t \cdot\left(1+O\left(e^{-\delta \psi}\right)\right)+ \\
& +O\left(\frac{e^{-\delta \psi}}{\log ^{2}(T)} \int_{T}^{2 T}\left(\int_{-\infty}^{\infty} \frac{\left|\zeta\left(\frac{1}{2}+\mathrm{i} t+\mathrm{i} v\right)\right|^{k}}{\left(\sigma-\frac{1}{2}\right)^{2}+v^{2}} \mathrm{~d} v\right)^{2} \mathrm{~d} t\right)
\end{aligned}
$$

By the same argument as in Lemma 3 the error term above is $\ll e^{-\delta \psi} \int_{T}^{2 T}\left|\zeta\left(\frac{1}{2}+\mathrm{i} t\right)\right|^{2 k} \mathrm{~d} t+T^{1-\varepsilon}$ which is $\ll e^{-\delta \psi} T(\log T)^{k^{2}}+T^{1-\varepsilon}$ by Theorem 1 . On the other hand the main term in (14) is equal to

$$
T \sum_{n \leqslant x} \frac{d_{k}(n)^{2} W(n)^{2}}{n^{2 \sigma}} \cdot\left(1+O\left(e^{-\delta \psi}\right)\right)=T \sum_{n \geq 1} \frac{d_{k}(n)^{2}}{n^{2 \sigma}} \cdot\left(1+O\left(e^{-\delta \psi}\right)\right),
$$

by an standard calculation. Since the main term above is $\asymp T(\log T / \psi)^{k^{2}}$ it dominates the error term. On combining these two estimates the claim follows.

\section{Proof of Proposition 1.}

Lemma 5. Assume the Riemann Hypothesis. Then, uniformly in $T \leqslant t \leqslant 2 T$ (with $T \geqslant T_{0}$ ) and $\sigma \geqslant \frac{1}{2}+1 / \log T$,

$$
\left|\zeta\left(\frac{1}{2}+\mathrm{i} t\right)\right|^{2 r} \leqslant T^{r(2 \sigma-1)} \int_{-\infty}^{\infty}|\zeta(s+\mathrm{i} v)|^{2 r} \cdot \frac{(1 / \pi)\left(\sigma-\frac{1}{2}\right) \mathrm{d} v}{\left(\sigma-\frac{1}{2}\right)^{2}+v^{2}}+O\left(\frac{1}{T}\right) .
$$

Proof. By Cauchy's formula and Lindelöf's Hypothesis (used to bound the integrals over horizontal lines)

$$
\zeta\left(s+\left(\sigma-\frac{1}{2}\right)\right)^{2 r}=\int_{-\infty}^{\infty} \zeta(s+\mathrm{i} v)^{2 r} \cdot \frac{(1 / \pi)\left(\sigma-\frac{1}{2}\right) \mathrm{d} v}{\left(\sigma-\frac{1}{2}\right)^{2}+v^{2}}+O\left(\frac{1}{T}\right),
$$

and by Lemma 1, $\left|\zeta\left(\frac{1}{2}+\mathrm{i} t+\mathrm{i} v\right)\right|^{2 r} \leqslant T^{r(2 \sigma-1)} \cdot\left|\zeta\left(s+\left(\sigma-\frac{1}{2}\right)\right)\right|^{2 r}$. Combining the two, and taking absolute values we obtain the claim.

Lemma 6. Let $w_{a}(v)=\frac{(1 / \pi) a}{a^{2}+v^{2}}$ for all $a>0$. Suppose that $f, g: \mathbb{R} \rightarrow \mathbb{R}^{+}$are two functions in $L^{2}\left(\mathbb{R} ; w_{a}(v) \mathrm{d} v\right)$. If

$$
f(x) \leqslant C \cdot g(x)+\frac{1}{4} \int_{-\infty}^{\infty} f(x+\mathrm{i} u) w_{a}(u) \mathrm{d} u,
$$

for some constant $C>0$, then,

$$
\int_{-\infty}^{\infty} f(x+\mathrm{i} v)^{2} \cdot w_{a}(v) \mathrm{d} v \leqslant 3 C^{2} \int_{-\infty}^{\infty} g(x+\mathrm{i} v)^{2} \cdot w_{a}(v) \mathrm{d} v .
$$


Proof. Note that

$$
\begin{aligned}
f(x)^{2} & \leqslant 2 C^{2} \cdot g(x)^{2}+\frac{1}{8} \cdot\left(\int_{-\infty}^{\infty} f(x+\mathrm{i} u) w_{a}(u) \mathrm{d} u\right)^{2} \\
& \leqslant 2 C^{2} \cdot g(x)^{2}+\frac{1}{8} \cdot \int_{-\infty}^{\infty} f(x+\mathrm{i} u)^{2} w_{a}(u) \mathrm{d} u \cdot \int_{-\infty}^{\infty} w_{a}(u) \mathrm{d} u,
\end{aligned}
$$

by the inequality $|a+b|^{2} \leqslant 2|a|^{2}+2|b|^{2}$ and Cauchy-Schwarz. Also $\int_{-\infty}^{\infty} w_{a}(u) \mathrm{d} u=1$. In the above, we let $x \mapsto x+\mathrm{i} v$ and integrate with respect to $w_{a}(v) \mathrm{d} v$ over the whole line $-\infty<v<\infty$. This gives,

$$
\int_{-\infty}^{\infty} f(x+\mathrm{i} v)^{2} w_{a}(v) \mathrm{d} v \leqslant 2 C^{2} \int_{-\infty}^{\infty} g(x+\mathrm{i} v)^{2} w_{a}(v) \mathrm{d} v+\frac{1}{8} \int_{-\infty}^{\infty} f(x+\mathrm{i} v)^{2} \cdot\left(w_{a} * w_{a}\right)(v) \mathrm{d} v,
$$

where $w_{a} * w_{a}(v)=\int_{-\infty}^{\infty} w_{a}(u) w_{a}(u-v) \mathrm{d} u$. Note that $\left(w_{a} * w_{a}\right)(v)=w_{2 a}(v)$ and that $w_{2 a}(v) \leqslant 2 w_{a}(v)$. Whence,

$$
\int_{-\infty}^{\infty} f(x+\mathrm{i} v)^{2} w_{a}(v) \mathrm{d} v \leqslant 2 C^{2} \int_{-\infty}^{\infty} g(x+\mathrm{i} v)^{2} w_{a}(v) \mathrm{d} v+\frac{1}{4} \int_{-\infty}^{\infty} f(x+\mathrm{i} v)^{2} w_{a}(v) \mathrm{d} v .
$$

Moving the last term to the left-hand side we obtain the claim.

Proof of Proposition 1. In the notation of Lemma 6, let $w(v):=w_{\sigma-\frac{1}{2}}(v)$. By a simple modification of Lemma 2,

$$
\zeta\left(s+\sigma-\frac{1}{2}\right)^{r}=\sum_{n \leqslant x} \frac{d_{r}(n) W(n)}{n^{s+\sigma-\frac{1}{2}}}+\frac{\theta y^{\frac{1}{2}-\sigma}}{\log (x / y)} \cdot \frac{1}{\pi} \int_{-\infty}^{\infty} \frac{|\zeta(s+\mathrm{i} v)|^{r} \mathrm{~d} v}{\left(\sigma-\frac{1}{2}\right)^{2}+v^{2}}
$$

where $x=T^{r / 2+2 \delta}$. Set $y=T^{r / 2+\delta}$ and $\sigma=\frac{1}{2}+\frac{4 / \delta}{\log T}$. By Lemma 1 and (15),

$$
\begin{aligned}
|\zeta(s)|^{r} & \leqslant e^{2 r / \delta} \cdot|\zeta(s+\sigma-1 / 2)|^{r} \\
& \leqslant e^{2 r / \delta} \cdot\left|\sum_{n \leqslant x} \frac{d_{r}(n) W(n)}{n^{s+\sigma-\frac{1}{2}}}\right|+\frac{1}{4} \int_{-\infty}^{\infty}|\zeta(s+\mathrm{i} v)|^{r} \cdot w(v) \mathrm{d} v
\end{aligned}
$$

Hence, by Lemma 6 (with $f(t)=|\zeta(\sigma+\mathrm{i} t)|^{r}$ and $\left.g(t)=\left|\sum_{n \leq x} \frac{d_{r}(n) W(n)}{n^{2 \sigma-1 / 2+\mathrm{i} t}}\right|\right)$,

$$
\int_{-\infty}^{\infty}|\zeta(s+\mathrm{i} v)|^{2 r} \cdot w(v) \mathrm{d} v \leqslant 3 e^{4 r / \delta} \int_{-\infty}^{\infty}\left|\sum_{n \leqslant x} \frac{d_{r}(n) W(n)}{n^{s+\sigma-\frac{1}{2}+\mathrm{i} v}}\right|^{2} \cdot w(v) \mathrm{d} v
$$

By Lemma 5 the left-hand side is $\geqslant e^{-8 r / \delta} \cdot\left|\zeta\left(\frac{1}{2}+\mathrm{i} t\right)\right|^{2 r}+O(1 / T)$ and the claim follows.

\section{REFERENCES}

[1] J. B. Conrey and A. Ghosh. A conjecture for the sixth power moment of the Riemann zeta-function. Internat. Math. Res. Notices, 76 (3):497 - 522, 1998.

[2] J. B. Conrey and S. M. Gonek. High moments of the Riemann zeta-function. Duke Math. J., 107 (3):577 $-604,2001$.

[3] J.-M. Deshouillers and H. Iwaniec. Power mean-values for Dirichlet's polynomials and the Riemann zeta-function. II. Acta. Arithm., 43:305 - 312, 1984.

[4] J.-M. Deshouillers and H. Iwaniec. Power mean values of the Riemann zeta-function. Mathematika, 29 (2):202-212, 1983.

[5] A. Diaconu, D. Goldfeld, and J. Hoffstein. Multiple Dirichlet series and moments of Zeta and $L$-functions. Compositio Math., 139 (3):297 - 360, 2003. 
[6] S. M. Gonek, C. P. Hughes, and J. P. Keating. A hybrid Euler-Hadamard product for the Riemann zeta-function. Duke Math. J., 136 (3):507 - 549, 2007.

[7] G. H. Hardy and J. E. Littlewood. Contributions to the theory of the Riemann zeta-function and the theory of the distribution of primes. Acta. Math., 41:119-196, 1918.

[8] D. R. Heath-Brown. Fractional moments of the Riemann zeta-function. J. London Math. Soc, 24 (2):65 $-78,1981$.

[9] D. R. Heath-Brown. Fractional moments of Dirichlet L-functions. Acta. Arithm., 145:397 - 409, 2010.

[10] C. P. Hughes and M. Young. The twisted fourth moment of the Riemann zeta function. J. Reine Angew. Math., 641:203 - 236, 2010.

[11] A. E. Ingham. Mean-value theorems in the theory of the Riemann zeta-function. Proc. London. Math. Soc., 27 (2):273 - 300, 1926.

[12] A. Ivić. On the moments of the Riemann zeta-function in short intervals. Hardy-Ramanujan J., 32:4$23,2009$.

[13] H. Iwaniec. On mean values for Dirichlet's polynomials and the Riemann zeta-function. J. London Math. Soc. (2), 22 (1):39 - 45, 1980.

[14] J. A. Gaggero Jara. Asymptotic mean square of the product of the second power of the Riemann zetafunction and a Dirichlet polynomial. PhD thesis, Rochester University, Rochester, NY, 1997.

[15] J. P. Keating and N. C. Snaith. Random matrices and L-functions. J. Phys. A., 36 (12):2859 - 2881, 2003.

[16] M. Radziwill and K. Soundararajan. Fractional moments of the Riemann zeta-function. I. , pre-print. 2010.

[17] K. Ramachandra. Some remarks on the mean-value of the Riemann zeta-function and other Dirichlet series. II. Hardy-Ramanujan J., 3:1-24, 1980.

[18] A. Selberg. On the remainder in the formula for $N(t)$, the number of zeros of $\zeta(s)$ in the strip $0<t<T$. Avh. Norske Vid. Akad. Oslo. I., no. 1:27 pp., 1944.

[19] K. Soundararajan. Moments of the Riemann zeta-function. Annals of Math., 170 (2):981 - 993, 2009.

[20] N. Watt. Kloosterman sums and a mean value for Dirichlet polynomials. J. Number. Th., 53:179-210, 1995.

Department of Mathematics, Stanford University, 450 Serra Mall, Bldg. 380, Stanford, CA 94305-2125

E-mail address: maksym@stanford.edu 